\title{
Human cytomegalovirus promotes the activation of TGF- $\beta 1$ in human umbilical vein endothelial cells by MMP-2 after endothelial mesenchymal transition
}

\author{
Gang Chen ${ }^{1, B-D, F}$, Xiaomei Yang ${ }^{2, B, F}$, Bangning Wang ${ }^{1, A, D-F}$, Ziping Cheng ${ }^{1, C, F}$, Ren Zha0 ${ }^{1, B, F}$ \\ ${ }^{1}$ Department of Cardiology, The First Affiliated Hospital of Anhui Medical University, Hefei, China \\ ${ }^{2}$ Department of Clinical Laboratory, The Second People's Hospital of Hefei, China \\ A - research concept and design; $\mathrm{B}$ - collection and/or assembly of data; $\mathrm{C}$ - data analysis and interpretation; \\ $D$ - writing the article; $E$ - critical revision of the article; $F$ - final approval of the article
}

Address for correspondence

Bangning Wang

E-mail: hfxnk33258@163.com

Funding sources

None declared

Conflict of interest

None declared

Received on May 31, 2018

Reviewed on August 3,2018

Accepted on May 7, 2019

Published online on November 28, 2019

Cite as

Chen G, Yang X, Wang B, Cheng Z, Zhao R. Human cytomegalovirus promotes the activation of TGF- $\beta 1$ in human umbilical vein endothelial cells by MMP-2 after endothelial mesenchymal transition. Adv Clin Exp Med. 2019;28(11): 1441-1450. doi:10.17219/acem/109199

DOI

10.17219/acem/109199

Copyright

Copyright by Author(s)

This is an article distributed under the terms of the

Creative Commons Attribution Non-Commercial License

(http://creativecommons.org/licenses/by-nc-nd/4.0/)

\begin{abstract}
Background. Human cytomegalovirus (HCMV) infection is one of the risk factors of cardiovascular disease; the most important pathological change is the change of vascular endothelial cell (VEC) function, but its mechanism is still unclear. Transforming growth factor $\beta 1$ (TGF- $\beta 1$ ) is an important cytokine associated with fibrosis; it can induce the occurrence of endothelial mesenchymal transition (EndMT) in VECS, which means endothelial cells acquire the characteristics and phenotypes of mesenchymal cells and secrete molecules associated with the deposition and remodeling of the extracellular matrix. Many in vivo and in vitro studies have shown that HCMV infection promotes the secretion and activation of TGF- $\beta 1$.
\end{abstract}

Objectives. This study aims to observe the changes of endothelial cells after HCMV infection and EndMT occurrence induced by TGF- $\beta 1$ and to explore the possible mechanism of HCMV infection in the pathogenesis of cardiovascular disease.

Material and methods. Immunofluorescence staining, reverse transcription polymerase chain reaction (RT-PCR), enzyme-linked immunosorbent assay (ELLSA), and immunoprecipitation methods were used in this study to analyze the changes in morphology and gene expression.

Results. We found that EndMT-related morphological and gene expression changes occurred in human umbilical vein endothelial cells (HUVECS) infected and uninfected with HCMV after treatment with TGF-B1. Human umbilical vein endothelial cells infected with HCMV, which are treated with TGF- $\beta 1$, can activate the extracellular potential TGF- $\beta 1$ by activating matrix metalloproteinase 2 (MMP-2).

Conclusions. Our findings provide a molecular basis for the association between HCMV infection, TGF- $\beta 1$ and cardiovascular disease.

Key words: TGF- $\beta 1$, cardiovascular disease, MMP-2, human cytomegalovirus (HCMV), endothelial mesenchymal transition 


\section{Introduction}

Human cytomegalovirus (HCMV) belongs to the $\beta$-herpesvirus family and is a double-stranded DNA virus. Human cytomegalovirus infection is very common all over the world, and the virus will remain in the body for the rest of one's life once infected. It has been found that HCMV is associated with a variety of cardiovascular diseases such as atherosclerosis (AS), coronary heart disease and hypertension. ${ }^{1-6}$ Human cytomegalovirus infection is also related to the dysfunction of vascular endothelial cells (VECs).$^{7-9}$ Antiviral drugs, including ganciclovir, can reduce the risk of a heart transplant-associated AS. ${ }^{10} \mathrm{Hu}$ man cytomegalovirus infection can also aggravate AS. ${ }^{11-14}$ However, the mechanism of HCMV infection in cardiovascular disease is still unclear.

Vascular endothelial cells are an important regulatory factor that can participate in a variety of physiological and pathological processes of cardiovascular disease. Recent studies have shown that endothelial dysfunction and its associated cardiovascular diseases can be induced through a process known as endothelial mesenchymal transition (EndMT). ${ }^{15}$ In the process of EndMT, endothelial cells (ECs) lose specific endothelial markers, such as vascular endothelial cadherin (VE-cadherin) and leukocyte differentiation antigen 31 (CD31), obtain the mesenchymal cell markers alpha smooth muscle actin ( $\alpha$-SMA), fibroblast specific protein 1 (keratin) and fibronectin, and acquire migratory, invasive and proliferative phenotypes. EndMTderived fibroblasts are increased in advanced atherosclerotic plaques, and there is a clear association between EndMT and the progression of AS. ${ }^{15-17}$ Endothelial mesenchymal transition is involved in the process of AS plaque formation and promotes the expression of cardiac fibroblasts and collagen. It is one of the major factors of myocardial fibrosis. ${ }^{18}$ The occurrence of EndMT is regulated by many cytokines; transforming growth factor $\beta 1$ (TGF- $\beta 1$ ) is the key cytokine for the development of EndMT and an important cytokine in vascular pathophysiology. ${ }^{19}$ Increased TGF- $\beta 1$ can promote the occurrence of cardiovascular diseases, such as pulmonary hypertension (PAH) and $\mathrm{AS}$, and blocking the expression of TGF- $\beta 1$ can inhibit the PAH process and the formation of unstable AS plaques. ${ }^{20}$

Cytomegalovirus infection can increase the expression of TGF- $\beta 1$, but TGF- $\beta 1$ is always in an inactive latent state. ${ }^{21}$ The activation factors of latent TGF- $\beta 1$ mainly contain protease (plasmin), metalloproteinase (MMPs), thrombospondin (TSP-1), $\alpha_{\mathrm{v}} \beta_{6}$, and $\alpha_{\mathrm{v}} \beta_{8}{ }^{22}$ In the HCMVinfected placenta, HCMV induces TGF- $\beta 1$ and collagen IV expression by $\alpha_{\mathrm{v}} \beta_{6} \cdot{ }^{23}$ Thus, HCMV-infected VECs may be involved in the pathogenesis of AS by inducing the expression or activation of TGF- $\beta 1$. In this study, we observed the changes of endothelial cells after HCMV infection and EndMT induced by TGF- $\beta 1$, and we explored the possible mechanism of HCMV infection in the pathogenesis of cardiovascular disease.

\section{Material and methods}

\section{Cells and virus}

Human umbilical vein endothelial cells (HUVECs), human embryonic lung fibroblasts (HELF) and mink lung epithelium cells were purchased from ATCC (Manassas, USA). Human umbilical vein endothelial cells were cultured with human endothelial serum-free medium containing bFGF (20 ng/mL), EFG (10 ng/mL) and human plasma fibronectin $(10 \mu \mathrm{g} / \mathrm{mL})$ (Life Technologies, Carlsbad, USA) at $37^{\circ} \mathrm{C}$. Human umbilical vein endothelial cells were randomly divided into the HCMV TR-infected group, uninfected group, HCMV-infected +raTGF- $\beta 1$ treatment group, and raTGF- $\beta 1$ treatment group. Human embryonic lung fibroblasts and mink lung epithelium cells were cultured with Dulbecco's modified Eagle's medium (DMEM) containing 10\% fetal bovine serum (Life Technologies) at $37^{\circ} \mathrm{C}$. Mink lung epithelium cells were used to detect TGF- $\beta 1$ activity.

The HCMV TR virus strain was cultured in HELF cells. The supernatant of the viral culture was centrifuged at $4^{\circ} \mathrm{C}$, $16,000 \times \mathrm{g}$ for $2 \mathrm{~h}$, and the virus was resuspended with human endothelial serum-free medium (SFM) and stored at $-80^{\circ} \mathrm{C}$. The virus was placed in a crosslinked chamber (Bio-Rad, Hercules, USA) and irradiated with $150 \mathrm{~mJ}$ UV rays to inactivate the virus. The titer of the virus was titrated in HELF cells by detection of early antigen fluorescent foci (DEAFF).

\section{Reagents}

Recombinant human active TGF- $\beta 1$ (raTGF- $\beta 1$ ), Quantikine human TGF- $\beta 1$ enzyme-linked immunosorbent assay (ELISA) kit and TGF- $\beta 1$ blocking antibodies were purchased from R\&D Systems (Minneapolis, USA); Luciferase assay kit and $\beta$-galactosidase detection kit were purchased from Promega Corp. (Madison, USA); RNeasy kit was purchased from Qiagen (Valencia, USA); RT ${ }^{2}$ First Strand Kit, SuperArray Human Extracellular Matrix PCR Array and human matrix metalloproteinase 2 (MMP-2) shRNA kit were purchased from SA Biosciences (Frederick, USA); GM6001, rabbit anti-MMP-2 antibody (AB19167), rabbit anti-MT3-MMP antibody (AB853) and mouse anti- $\alpha v \beta 6$ blocking antibody (MAB2077Z) were purchased from Merck Millipore (Billerica, USA); antiGFP antibody, AlexaFluor conjugated phalloidin and secondary antibodies, and SuperScript III kit were purchased from Invitrogen (Carlsbad, USA); Cytogam was purchased from CSL Behring (King of Prussia, USA); Nucleofector device and transfection kit $\mathrm{V}$ were purchased from Amaxa (Gaithersburg, USA); RT-PCR primers were purchased from Applied Biosystems (Waltham, USA); fibronectin (Hs.01549976_m1), MMP-9(Hs.00957562_m1), ADAMTS1 (Hs.00199608_m1), TGF-ß1 (Hs.00932734_m1), collagen 5A1 (Hs.00609088_m1), MMP-2 (Hs.01548733_ m1), thrombospondin-1 (Hs.00170236_m1), 18S RNA (part 
\#4333760-0904029), mouse anti-MMP-2, mouse antiTIMP-2, and rabbit anti-MT1-MMP polyclonal antisera were purchased from Abcam (Cambridge, UK); mouse anti HCMV, IE1 and p52 monoclonal antibodies were provided by Dr. Gan (Anhui Medical University, Hefei, China).

\section{Detection of virus titer}

Human embryonic lung fibroblasts and HUVECs were inoculated in 6-well plates; HCMV was inoculated in the cells according to multiplicity of infection $(\mathrm{MOI})=1$ and the cells were cultured at $37^{\circ} \mathrm{C}$ for $1 \mathrm{~h}$. They were washed with phosphate-buffered saline (PBS) 3 times, and culture medium was added into them; the supernatant and cells were collected daily for 6 days and then stored in $-80^{\circ} \mathrm{C}$. The cells were resuspended with $0.5 \mathrm{~mL}$ of PBS, and the titer was detected using DEAFF after serial dilution. The titer of the supernatant was also detected using DEAFF after serial dilution.

\section{Immunofluorescence staining}

Human cytomegalovirus TR virus was inoculated in the HELF and HUVECs in 24-well plates on coverslips according to MOI = 1 with or without recombinant active TGF- $\beta 1$ (raTGF- $\beta 1,15 \mathrm{ng} / \mathrm{mL}$ ) treatment for 6 days. The cells were fixed with $4 \%$ paraformaldehyde and permeabilized with $0.1 \%$ Triton X-100. They were incubated at $4^{\circ} \mathrm{C}$ overnight after appropriately diluted primary antibodies were added. Then, they were washed and incubated at room temperature for $1 \mathrm{~h}$ with AlexaFluor 488or AlexaFluor 594-labeled secondary antibodies. Then, they were incubated at room temperature for $15 \mathrm{~min}$ with AlexaFluor 488-labeled phalloidin and 4',6-diamidino2-phenylindole (DAPI), and the slides were mounted after washing and observed under a fluorescence microscope (Olympus Fluoview BX51, Center Valley, USA).

\section{Western blotting analysis}

The cells were harvested and lysed with radioimmunoprecipitation assay (RIPA) lysis buffer (PBS containing 1\% NP40, 0.1\% SDS, 5 mM EDTA, 0.5\% sodium deoxycholate, $1 \mathrm{mM}$ sodium orthovanadate, and protease inhibitors) on ice for $30 \mathrm{~min}$ with shaking at $12,000 \mathrm{rpm} / \mathrm{min}$. Total cellular protein was collected, and the concentration was determined with a BCA Kit. Equal amounts of protein $(25 \mu \mathrm{g} /$ well) were separated using 10\% SDS-PAGE electrophoresis. Then, it was electrotransferred to the polyvinylidene fluoride (PVDF) membrane. After the transfer, the PVDF membrane was rinsed with TBS for 10-15 min, placed in TBS/T blocking buffer containing $5 \%$ (w/v) skimmed milk powder and shaken at room temperature for $2 \mathrm{~h}$. It was incubated at $4^{\circ} \mathrm{C}$ overnight after adding primary antibodies at the appropriate dilution. Then, the membrane was washed with TBST 3 times (for 5 min each time). The membrane was incubated at $37^{\circ} \mathrm{C}$ for $1 \mathrm{~h}$ with HRP labeled secondary antibody $(1: 10,000)$, diluted with TBST containing $0.05 \%(\mathrm{w} / \mathrm{v})$ skimmed milk powder. The gel was developed with ECL (Perkin-Elmer Inc., Waltham, USA) for $5 \mathrm{~min}$. The protein bands were quantified using an Imagequant LAS4000 (GE Healthcare, Tokyo, Japan).

\section{RNA extraction and RT-PCR}

Total RNA was extracted from different groups using the RNeasy kit according to the manufacturer's manual. Their concentration and purity were detected with an Agilent 2100 Bioanalyzer (Agilent Technologies Inc., Santa Clara, USA). A total of $1 \mu \mathrm{g}$ of RNA was subjected to reverse transcription using the RT ${ }^{2}$ First Strand Kit. Reverse transcription PCR was performed using the FastStart Universal SYBR Green Master (Thermo Fisher Scientific, Waltham, USA). Real-time polymerase chain reaction (RT-PCR) primers were purchased from Applied Biosystems. Reaction parameters were $95^{\circ} \mathrm{C}$ for $45 \mathrm{~s}, 95^{\circ} \mathrm{C}$ for $5 \mathrm{~s}$ and $60^{\circ} \mathrm{C}$ for $30 \mathrm{~s}$, with 40 cycles. The relative expression of genes was calculated using 18S RNA as control. The expression levels of TIMP-2, MT1-MMP and MT3-MMP microRNA (mRNA) in different treated HUVECs were detected using RT-PCR methods; they were performed using Platinum ${ }^{\text {TM }}$ Taq Green Hot Start DNA Polymerase (Life Technologies) according to the instructions. The primers were as follows: TIMP-2 (F:5'-GAGCGAGAAGGAGGTGGATTCCGGG-3'; R: 5'-ATGTCAAGAAACTCCTGCTTCGGGGG-3'); MT1-MMP (F:5'-GGATACCCAATGCCCATTGGCCA-3'; R: 5'-CATTGGGCATCCAGAAGAGAGC-3'); MT3-MMP (F: 5'-ACAGTCTGCGGAACGGAGCAG-3'; R: 5'-GTCAATTGTGTTTCTGTCCAC-3'); $\beta$-actin (F:5'-TGAGGATGTCACGGTTCCAG-3'; R: 5'-GTCACCTTCACCGTTCCAGT-3').

\section{TGF- $\beta 1$ content detection}

Human umbilical vein endothelial cells were seeded in a 24 -well plate $\left(2 \times 10^{4}\right.$ cells/well $)$ and 6 -well plate $\left(1 \times 10^{5} \mathrm{cells} / \mathrm{well}\right)$, and EndMT in cells was induced after they were treated with raTGF- $\beta 1$ for $48 \mathrm{~h}$. Then, HCMV was inoculated in the cells according to $\mathrm{MOI}=1$, and HUVECs without EndMT or HCMV infection were used as the control. Human umbilical vein endothelial cells were infected according to $\mathrm{MOI}=2,4,6,8$, and 10 to analyze $\mathrm{HCMV}$ infection dose; then, they were cultured at $37^{\circ} \mathrm{C}$ for $1 \mathrm{~h}$ and then washed 3 times, and the raTGF- $\beta 1$ was removed. Then, they were cultured with fresh medium for $24 \mathrm{~h}$, and the TGF- $\beta 1$ content in the culture supernatant was detected. The activation of luciferase was determined according to reference. ${ }^{24}$ The activated TGF- $\beta 1$ and total TGF- $\beta 1$ in the cell culture supernatant was quantified using the Quantikine human TGF- $\beta 1$ ELISA kit and an Ebioscience ELISA kit according to the kit instructions, respectively. 


\section{ELISA assay}

Transforming growth factor $\beta 1$ neutralizing antibody $(0.5 \mu \mathrm{g} / \mathrm{mL}, 1 \mu \mathrm{g} / \mathrm{mL}, 2 \mu \mathrm{g} / \mathrm{mL}$, and $4 \mu \mathrm{g} / \mathrm{mL})$ was added to HUVECs and they were treated with raTGF- $\beta 1$ (final concentration $0.6 \mathrm{nM}$ ) for $48 \mathrm{~h}$. Human cytomegalovirus TR virus was inoculated in the cells according to $\mathrm{MOI}=1$, and they were cultured at $37^{\circ} \mathrm{C}$ for $1 \mathrm{~h}$. Then, the cells were washed 3 times and cultured with fresh medium for $24 \mathrm{~h}$. The TGF- $\beta 1$ content in the culture supernatant was detected using bioassay of the reporter gene in mink lung epithelial cells. GM6001 (0.5 nM), aprotinin (200 mg/mL), anti-thrombospondin-1 (25 ng/ml) and anti$\alpha_{v} \beta_{6}(10 \mathrm{mg} / \mathrm{mL})$ were added to them before raTGF- $\beta 1$ treatment for $1 \mathrm{~h}$ to analyze the effect of different inhibitors on the activation of TGF- $\beta 1$.

\section{Immunoprecipitation with MMP-2 antibody}

Human umbilical vein endothelial cells were infected with HCMV or treated with raTGF- $\beta 1$ according to the above method. The cells were harvested using centrifugation and lysed with pre-cooled RIPA lysis buffer containing protease inhibitor. Matrix metalloproteinase 2 antibody was added to the cells, which were incubated at $4^{\circ} \mathrm{C}$ overnight. Then, the protein A-agarose was added and incubated with them, and they were lysed with precooled RIPA lysis buffer. The cells were resuspended, SDSPAGE loading buffer was added to them, and the resulting solution was boiled. Electrophoresis was carried out with 8\% SDS-PAGE gel. TIMP-2, MT1-MMP and MT3-MMP were detected in the cell lysate before and after immunoprecipitation using the western blot method.

\section{MMP-2 shRNA transfection}

Matrix metalloproteinase 2 shRNA and its control plasmids were transfected into HUVECs using the Amaxa Cell Line Nucleofector Kit. The cells were treated with raTGF- $\beta 1$ when a clear green fluorescence was observed in the cells under fluorescence microscopy, and HCMV was inoculated according to $\mathrm{MOI}=1$. The supernatant and cells were collected with centrifugation. The TGF- $\beta 1$ content in the supernatant was detected using a bioassay of the reporter gene in mink lung epithelial cells. Matrix metalloproteinase 2, GFP and $\beta$-actin expression levels in the precipitate were detected using western blot and RT-PCR methods.

\section{Statistical analysis}

The data was expressed as the mean values \pm standard error of the mean (SEM). All analyses were conducted using Prism v. 5.0 software (GraphPad, San Diego, USA). To compare 2 groups, we used the Student's t-test.
To compare more than 2 groups, we used one-way analysis of variance (ANOVA) or two-way ANOVA. P-values $<0.05$ were considered an indicator of a significant difference.

\section{Results}

\section{HCMV can proliferate in HUVECs but is not affected by raTGF- $\beta 1$}

A logarithmic increase in the number of progeny viruses was observed in the supernatants and HELF cells, which indicated that HELF supports the proliferative infection HCMV TR (Fig. 1A). Human umbilical vein endothelial cells also support the proliferative infection of HCMV $\mathrm{TR}$, but the proliferation curve is linear; the progeny virus proliferation was detected only in cells (Fig. 1B). The proliferation curves of HCMV in raTGF- $\beta 1$ treated and untreated HUVECs were not different (Fig. 1C), which was different from the results of HELF cells. Indirect immunofluorescence results showed that HCMV p52 could be expressed in the nucleus of HUVECs, and the distribution was similar to that in HELF (Fig. 1D). Human cytomegalovirus $\mathrm{p} 52$ is a coenzyme of DNA polymerase, and p52 expression indicates that HCMV enters proliferative infection.

\section{HCMV can infect HUVECs with EndMT induced by TGF- $\beta 1$}

Indirect immunofluorescence results showed that the cytoskeletal structure of HUVECs infected with HCMV was oval, which was the same as that of the control group. However, the cytoskeleton changed obviously after they were treated with raTGF- $\beta 1$, and the elongated mesenchymal phenotype appeared to form a parallel actin stress fiber, while the oval endothelial morphology disappeared. These results showed that HCMV infection can cause EndMT-like morphological changes in HUVECs in the presence of raTGF- $\beta 1$ (Fig. $2 \mathrm{~A}$ ).

Western blot results showed that VE-cadherin was expressed in the HCMV-infected group and the control group, but $\alpha$-SMA was not expressed in these groups, which is the phenotype of endothelial cells. However, $\alpha$-SMA was expressed in the raTGF- $\beta 1$ treatment group and the HCMV and raTGF- $\beta 1$ co-treatment group, while VE-cadherin was not expressed in these groups, which matches the phenotype of mesenchymal cells. In all raTGF- $\beta 1$ treatment groups, p-SMAD2 could be detected, but p-SMAD2 was not expressed in the HCMV infection alone group. This suggested that TGF- $\beta 1$ induced EndMT in HCMV-infected HUVECs also through SMAD2 signaling pathways. Human cytomegalovirus infection could induce the expression of $\alpha$-SMA and p-SMAD2 without VE-cadherin expression in the presence of raTGF- $\beta 1$; these changes were 


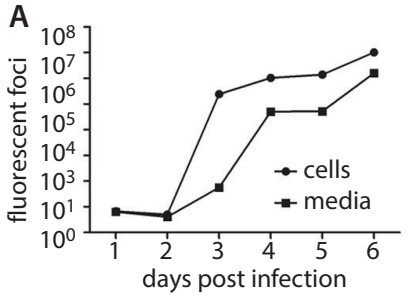

D

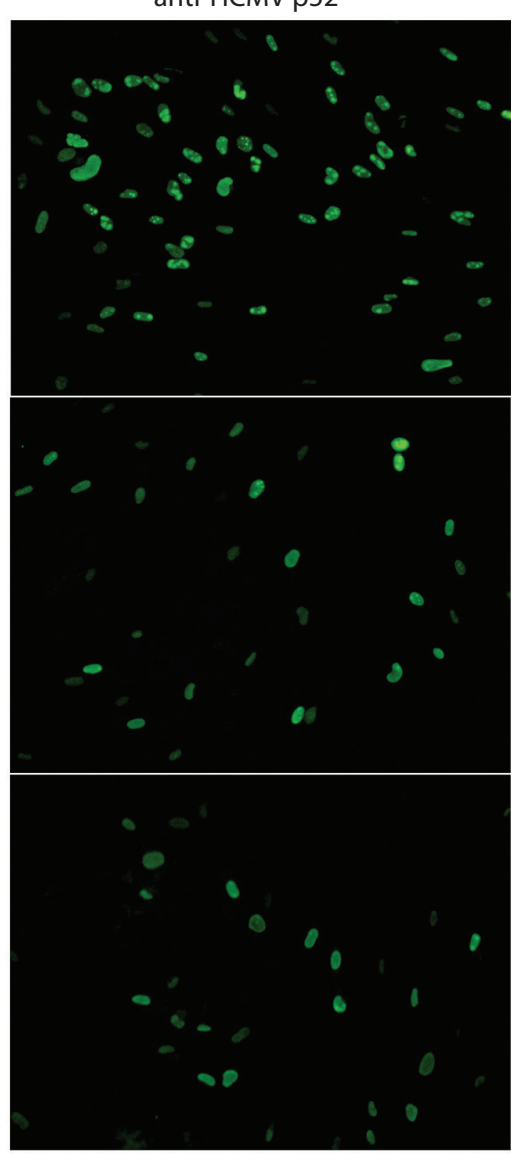

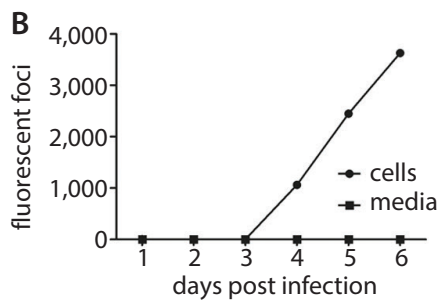

DAPI

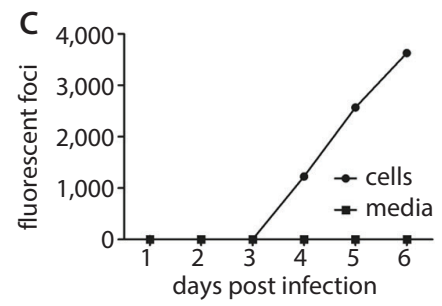

merged

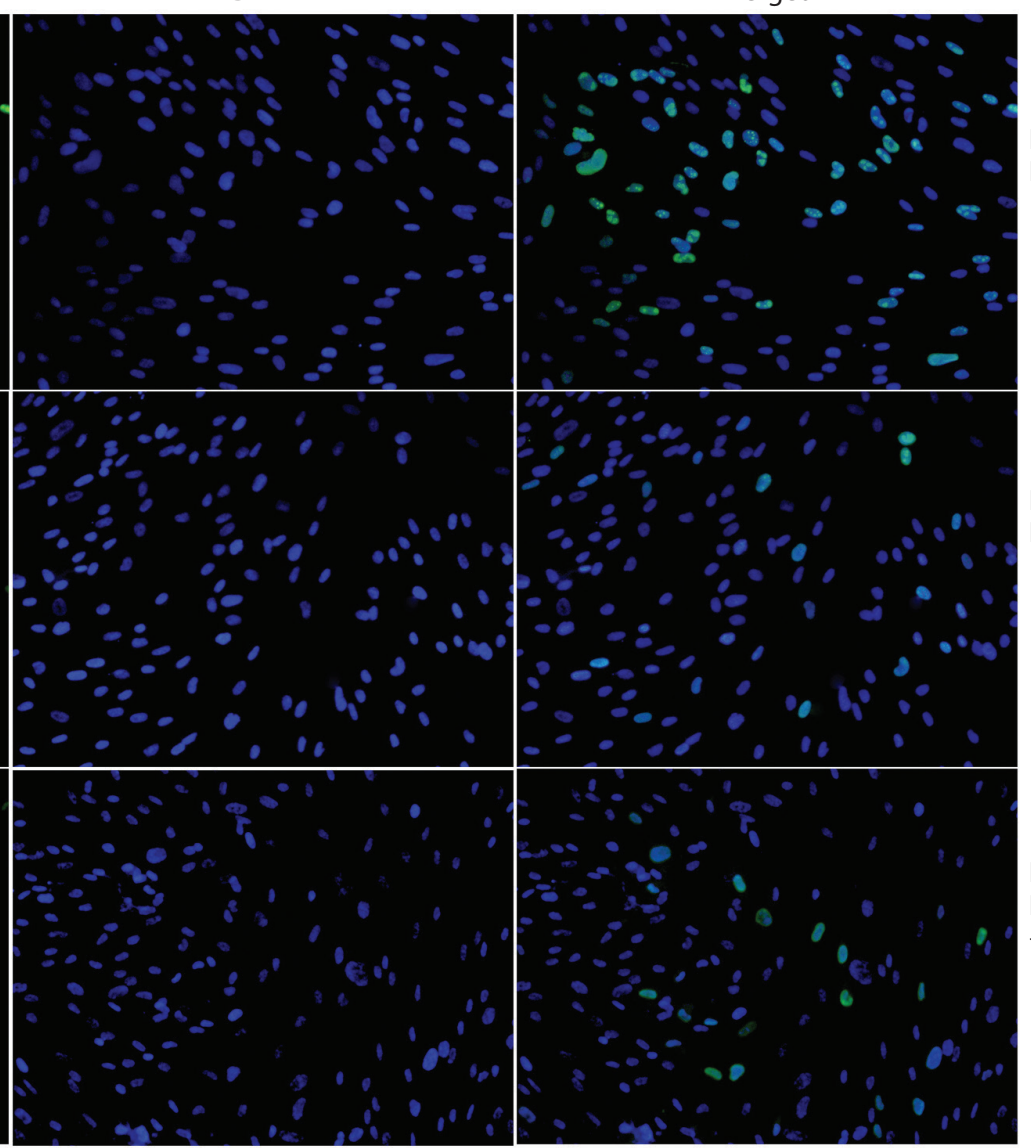

HCMV-infected

HELF

HCMV-infected HUVECS

HCMV-infected

HUVECS

+ raTGF- $\beta 1$

Fig. 1. The proliferation of HCMV in HUVECS

A - the titer of HCMV in HCMV-infected HELF cells and their culture supernatant; B - the titer of HCMV in HCMV-infected HUVECs and their culture supernatant; $\mathrm{C}$ - the titer of HCMV in raTGF- $\beta 1$-treated and HCMV-infected HUVECs and their culture supernatant; D - indirect immunofluorescence results of HCMV p52 expression in HELF cells, HUVECs and raTGF- $\beta 1$-treated HUVECs.

consistent with the phenotypic changes of HUVECs with EndMT (Fig. 2B).

Reverse transcription PCR results showed that the mRNA expression changes of fibrosis matrix protein in HCMV-infected HUVECs were less than 10 times compared with those of the control group, which suggested that there were no significant changes in cell phenotypes; this was consistent with morphological observations. In contrast, the mRNA expression levels of fibronectin, MMP-9 and $\alpha_{\mathrm{v}}$ integrin were significantly increased in the raTGF- $\beta 1$ treatment groups. The expression level of TIMP-2 in the HCMV-infected group was higher than that in the raTGF- $\beta 1$ treatment group and the HCMV and raTGF- $\beta 1$ co-treatment group. The expression levels of ADAMTS1, TGF- $\beta 1, \beta$-catenin, collagens, MMPs, TIMP-1, and thrombospondins in the HCMV and raTGF- $\beta 1$ co-treatment group were higher than that of the HCMV-infected group and the raTGF- $\beta 1$ treatment group. These results suggested that HCMV infection upregulates the expression of many fibrosis molecules in HUVECs with EndMT induced by raTGF- $\beta 1$ (Fig. 2C).

\section{HCMV could induce the activation of TGF- $\beta 1$ in HUVECs with EndMT}

The luciferase reporter gene assay and ELISA assay showed similar results. The luciferase reporter gene assay was more sensitive. The results showed that only a small amount of activated TGF- $\beta 1$ could be detected in the culture supernatant of HCMV TR-/raTGF- $\beta 1-$ HUVECs and HCMV TR+/raTGF- $\beta 1-$ HUVECs, which suggested that HCMV infection did not induce the upregulation 
A

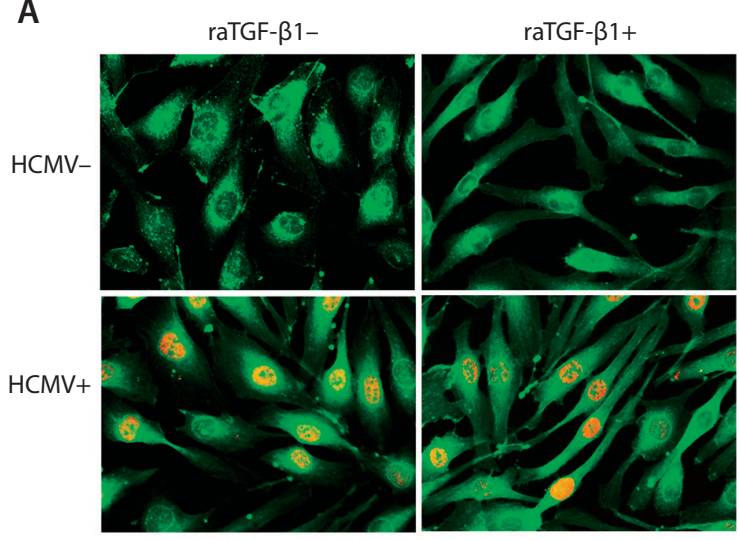

B

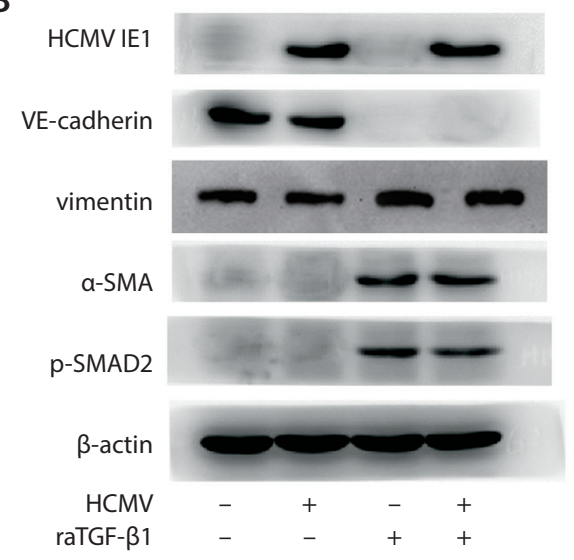

C

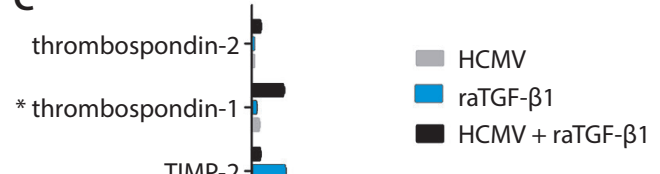

Fig. 2. HCMV could infect HUVECs with EndMT induced by TGF- $\beta 1$

A - indirect immunofluorescent staining results; B - western blotting results of HCMV IE1, VE-cadherin, vimentin, and a-SMA; C - RT-PCR results of extracellular matrix-related genes expression

A

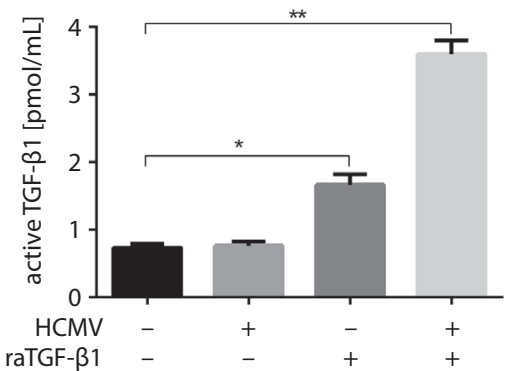

C

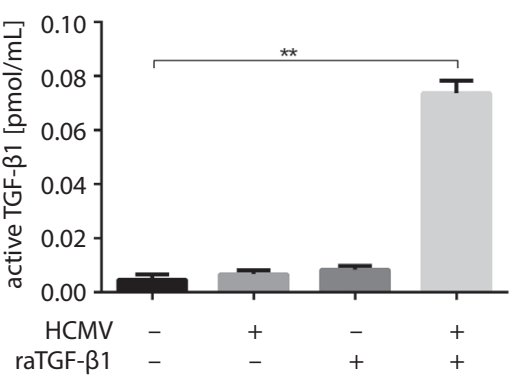

B

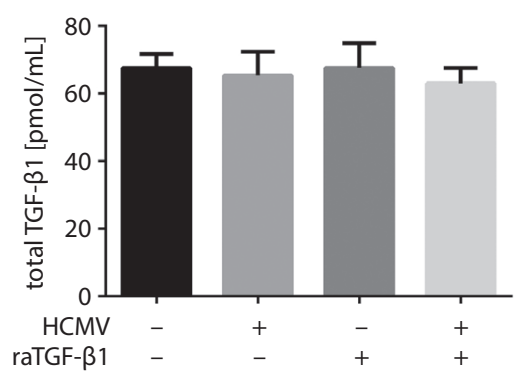

D

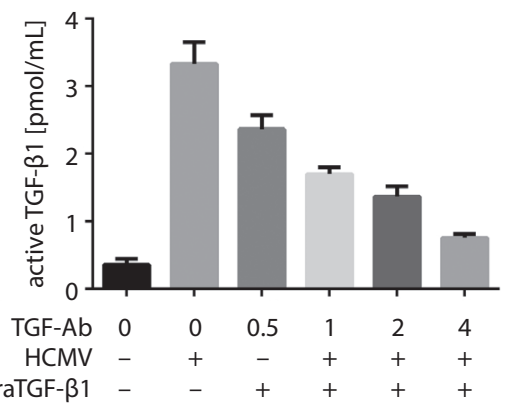

Fig. 3. HCMV could induce the activation of TGF- $\beta 1$ in HUVECs with EndMT

A - the content of activated TGF- $\beta 1$ in supernatant of cell culture detected using luciferase reporter gene assay; $B$ - the content of total TGF- $\beta 1$ in supernatant of cell culture detected using Quantikine human TGF- $\beta 1$ ELISA kit; C - the content of activated TGF- $\beta 1$ in supernatant of cell culture detected using ELISA assay; D - TGF- $\beta 1$ antibody can inhibit the activation of TGF- $\beta 1$ 
of TGF- $\beta 1$ activation in HUVECs (Fig. 3A,C). The content of activated TGF- $\beta 1$ in the culture supernatant was similar in HCMV TR-/raTGF- $\beta 1+$, HCMV TR-/raTGF- $\beta 1-$ and HCMV TR+/raTGF- $\beta 1-$ HUVECs. These results suggested that activated TGF- $\beta 1$ could not be upregulated in HUVECs with EndMT induced by exogenous raTGF- $\beta 1$ (Fig. 3A,C). The content of activated TGF- $\beta 1$ in culture supernatant significantly increased in HCMV TR+/raTGF- $\beta 1+$ HUVECs. These results showed that only HCMV infection or raTGF- $\beta 1$ treatment could not induce new activation of TGF- $\beta 1$ in HUVECs; the new activated TGF- $\beta 1$ could be produced when HUVECs with EndMT were infected with HCMV (Fig. 3B). The content of activated TGF- $\beta 1$ gradually decreased in the supernatant of cell culture with the increase of blocking antibodies (Fig. 3D).

\section{The amount of newly synthesized activated TGF- $\beta 1$ was positively correlated with the infective dose of TGF- $\beta 1$ and HCMV}

As shown in Fig. 4A, the amount of activated TGF- $\beta 1$ in the supernatant increased with the increase of the raTGF- $\beta 1$ amount, but there was no significant change in total TGF- $\beta 1$ among different groups, which suggested that HCMV-infected HUVECs with EndMT could stimulate the cells to secrete activated TGF- $\beta 1$ in an autocrine manner. Figure $4 \mathrm{~B}$ shows that the content of activated TGF- $\beta 1$ in the supernatant of cell culture increased with the increase of HCMV MOI. Ultraviolet inactivated HCMV did not upregulate the amount of activated
TGF- $\beta 1$ (Fig. 4C). These results suggested that the binding of HCMV and HUVECs with EndMT did not result in the production of activated TGF- $\beta 1$, and replication and proliferation of viruses are required in cells.

\section{MMP-2 is involved in the upregulation and activation of TGF- $\beta 1$ in VECs with EndMT induced by HCMV infection}

We found that the activated TGF- $\beta 1$ decreased by $60 \%$ when the HUVECs were treated with GM6001 ( $<<0.01$ ), and the activated TGF- $\beta 1$ decreased by $9 \%$ when the HUVECs were treated with aprotinin $(\mathrm{p}>0.05)$. Other blocking agents had no effect on the production of activated TGF- $\beta 1$. These results suggested that $\mathrm{MMP}$ and serine proteases may be involved in the upregulation of activated TGF- $\beta 1$ in HUVECs with EndMT infected by HCMV (Fig. 5A). Immunoprecipitation results showed that MMP-2 was bound with TIMP-2 and MT3-MMP, but not with MT1-MMP. These results suggested that HCMV infection may activate MMP-2. Matrix metalloproteinase 2 formed a membrane associated complex with TIMP-2 and MT3-MMP in HUVECs with EndMT, which promoted the formation and secretion of TGF- $\beta 1$ (Fig. 5C,E). Reverse transcription PCR and western blotting results showed that the mRNA and protein expression of MMP-2 decreased significantly after short hairpin RNA (shRNA) transfection (Fig. 5B,D), and the amount of activated TGF- $\beta 1$ in the cell supernatant was also significantly reduced (Fig. 5F). These results indicated that HCMV infection induced the activation of TGF- $\beta 1$ by activating MMP-2 in HUVECs with EndMT cells.
A

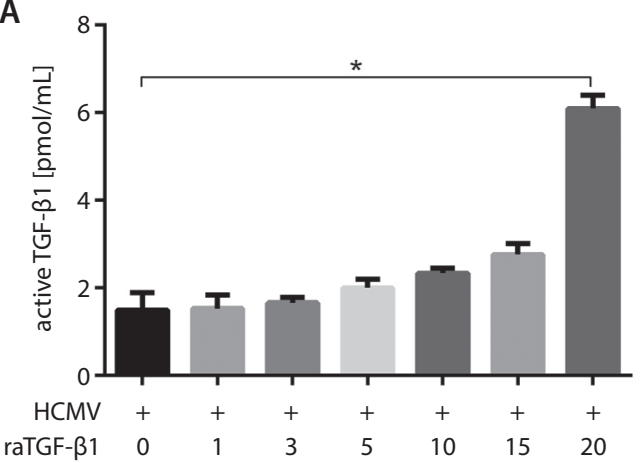

B

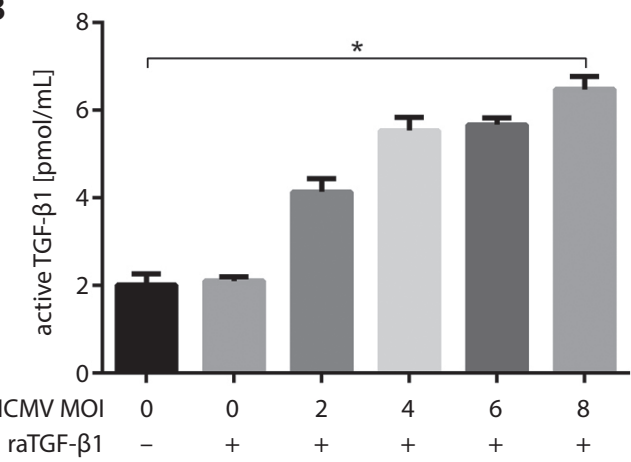

C

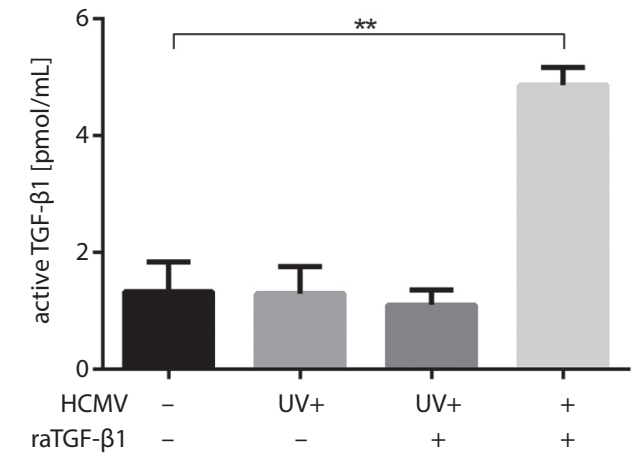

Fig. 4. The amount of newly synthesized activated TGF- $\beta 1$ was positively correlated with the infective dose of TGF- $\beta 1$ and HCMV

$A$ - the amount of activated TGF- $\beta 1$ in the supernatant increased with the increase of the raTGF- $\beta 1$ amount; $B$ - the content of activated TGF- $\beta 1$ in the supernatant increased with the increase of HCMV MOI; $\mathrm{C}$ - ultraviolet-inactivated HCMV did not upregulate the amount of activated TGF- $\beta 1$. 
A

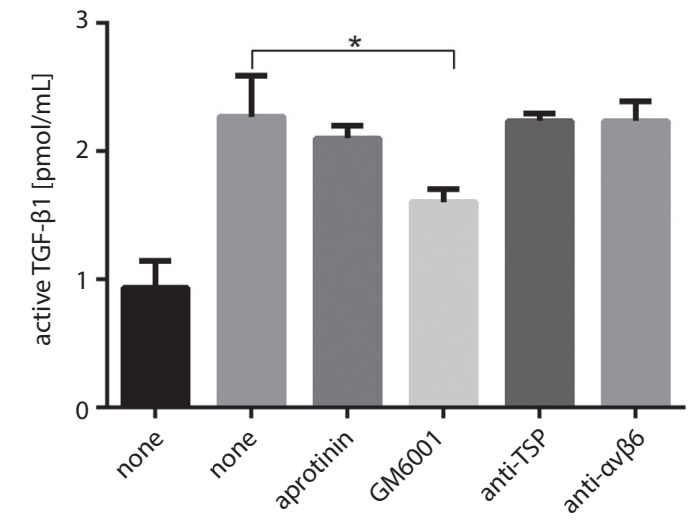

$\begin{array}{rrrrrrr}\text { HCMV } & - & + & + & + & + & + \\ \text { raTGF- } \beta 1 & - & - & + & + & + & +\end{array}$

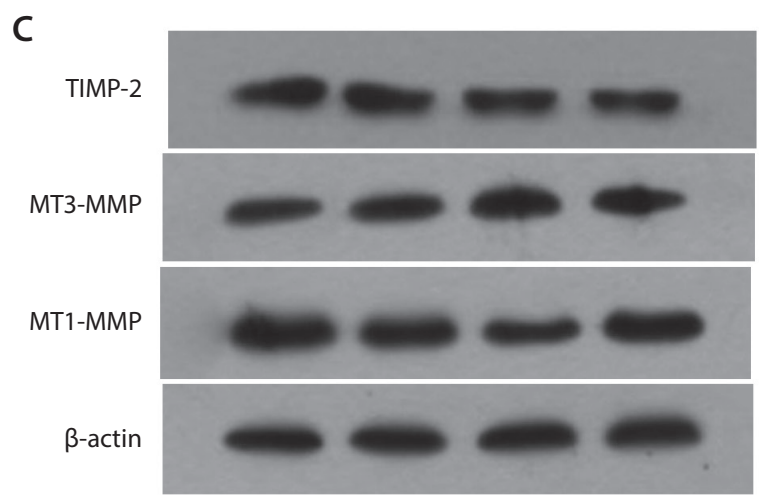

E MMP-2

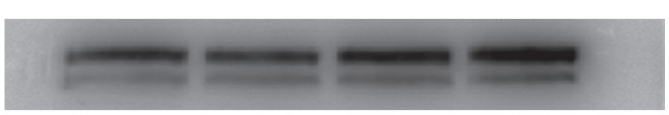

TIMP-2

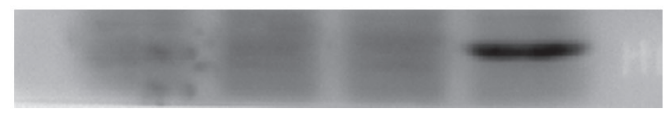

MT3-MMP

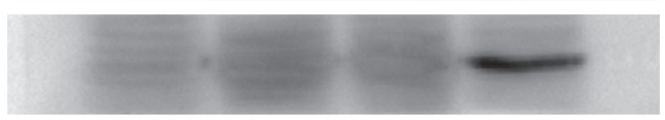

MT1-MMP

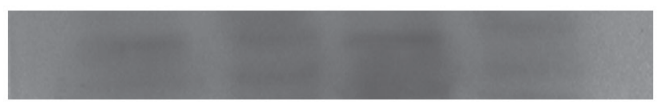

$\mathrm{HCMV}$
raTGF- $\beta 1$
B

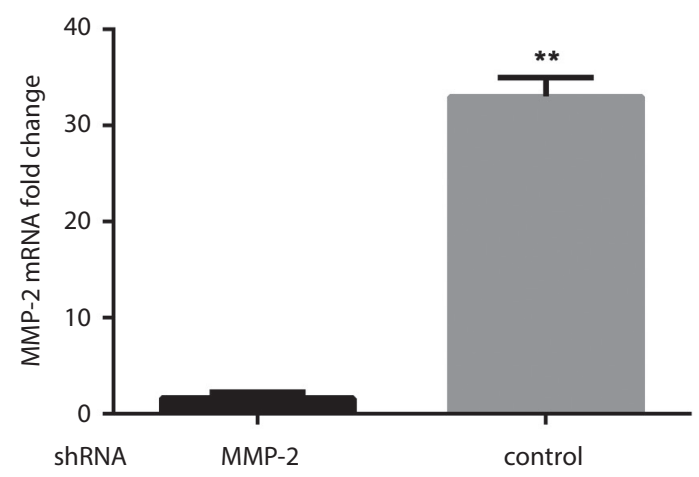

D

MMP-2

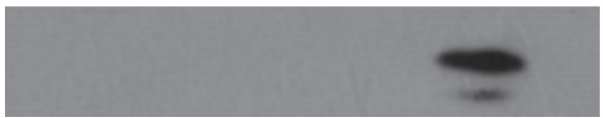

GFP

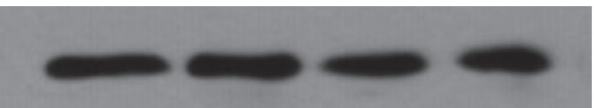

$\beta$-actin

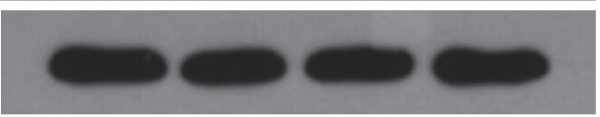

ShRNA MMP-2 control $\begin{array}{rlll}\mathrm{raTGF}-\beta 1 & - & + & +\end{array}$

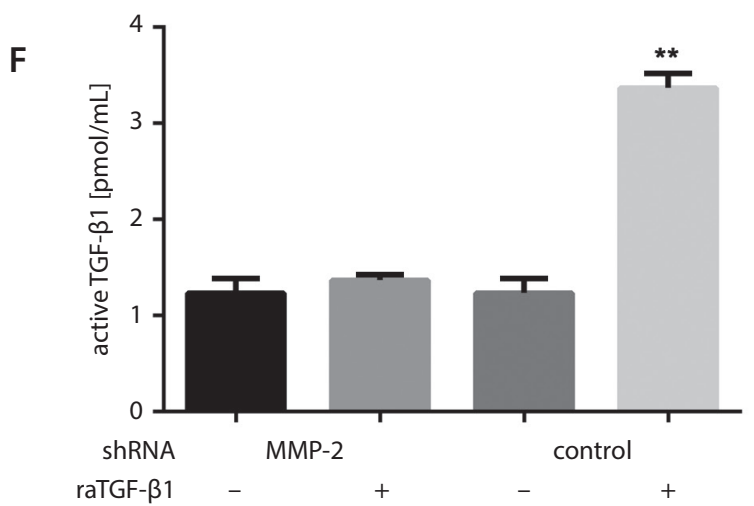

Fig. 5. MMP-2 is involved in the upregulation and activation of TGF- $\beta 1$ in HUVECs with EndMT induced by HCMV infection

A - the effect of different blocking agents on the production of activated TGF- $\beta 1$, GM6001 could significantly inhibit the activation of TGF- $\beta 1$; B - RT-PCR results showed that MMP-2 mRNA expression was downregulated after shRNA transfection; C - western blotting and RT-PCR results of TIMP-2, MT3-MMP and MT1-MMP proteins; D - western blotting results of MMP-2 after shRNA transfection; E - immunoprecipitation results showed that MMP-2, TIMP-2 and MT3-MMP could form complexes; F - transfection of HUVECs with shRNA vector inhibited the activation of TGF- $\beta 1$ induced by HCMV.

\section{Discussion}

Endothelial mesenchymal transition participates in many physiological and pathological processes of the cardiovascular system, and endothelial cells are involved in the formation of cardiac valves through EndMT in heart development. ${ }^{25}$ Endothelial mesenchymal transition is also involved in the formation of embryonic vascular systems. ${ }^{26}$ It is shown that EndMT is related to myocardial fibrosis; the production and accumulation of collagen and cardiac fibroblasts are related to the process of EndMT in patients with myocardial fibrosis. ${ }^{27}$ Endothelial mesenchymal transition can promote perivascular fibrosis in diabetic mice. ${ }^{28}$ Chen et al. found that TGF- $\beta$-induced EndMT could promote plaque 
growth and increase plaque thickness by increasing the deposition of extracellular matrix and enhancing the expression of adhesion molecules such as VCAM-1 and ICAM-1 in endothelial cells. ${ }^{18}$ Pulmonary arterial hypertension (PHA) is a chronic disease caused by accumulation of fibroblasts and the thickening or narrowing of the arterial wall. ${ }^{29} \mathrm{Re}-$ cent studies have demonstrated the presence of EndMT in the pulmonary arteries of hypertension, and the accumulation of transformed cells produced by EndMT increases blood pressure and results in cardiac dysfunction and heart failure. ${ }^{30}$ Studies have confirmed that TGF- $\beta 1$ is involved in the pathogenesis of myocardial fibrosis, AS and PHA. Human cytomegalovirus infection may promote the expression of TGF- $\beta 1$ or increase its activity, and it may also be associated with cardiovascular disease.

In this study, we found that HCMV could infect HUVECs. Human cytomegalovirus infection did not affect the expression level of TGF- $\beta 1$ in HUVECs; however, the autocrine of activated TGF- $\beta 1$ in HUVECs could be enhanced by MMP cascade reaction and promote the EndMT of HUVECs. These could promote the development of cardiovascular disease. The autocrine phenomenon helps us understand why elevated levels of TGF- $\beta 1$ are observed in patients with cardiovascular disease or animal models after infection with HCMV; it also helps promote the pathological process of fibrosis in vivo. ${ }^{19}$ It was shown that MMPs were involved in fibrosis-related cardiovascular diseases by degrading the basement membrane, enhancing cell motility, activating cell growth factors, and regulating cell adhesion molecules. ${ }^{31}$ It is believed that the regulation of MMP activity will become a new potential therapeutic method for cardiovascular disease. However, the effect of MMPs on cardiovascular disease with HCMV infection has not been further studied. In this study, we found that the trimer formed by MMP-2, MT3-MMP and TIMP-2 can promote the activation of TGF- $\beta 1$ in HUVECs infected by HCMV, and this may be the mechanism of TGF- $\beta 1$ activation induced by HCMV infection in VECs.

Epidermal growth factor (EGF) can enhance the migration phenotype of HK-2 cells induced by TGF- $\beta 1$, and synergistically increase the expression of MMP-9. ${ }^{32}$ Interestingly, we found that HCMV infection caused fibrosis in HUVECs after EndMT; HUVECs with endothelial phenotype infected by HCMV could not significantly increase the expression of TGF- $\beta 1$. This suggests that in normal circumstances, HCMV infection of VECs does not cause the appearance of fibroblasts, which is also consistent with the presence of no cardiovascular changes in asymptomatic patients with HCMV infection.

The effect of HCMV on the extracellular matrix and fibrosis has been verified in heart and kidney transplantation models of infected cytomegalovirus rats. ${ }^{33,34} \mathrm{Cy}-$ tomegalovirus infection can upregulate the expression of a large number of molecules associated with fibrosis and angiogenesis, and HCMV infection could also activate TGF- $\beta 1$ by the integrin mediated pathway. These mechanisms suggest that HCMV infection of the placenta can alter the extracellular matrix, allow HCMV transplacental translocation, and promote congenital infection. Our results support the possibility that HCMV infection may alter the extracellular matrix in inflammatory conditions. Therefore, under the condition of decreased immune status with local inflammation and EMT caused by TGF- $\beta 1$ in the host cells, latent infection of HCMV may be involved in the progression of vascular fibrosis by reactivation. These conditions may exist simultaneously in cardiac transplantation or cardiac bypass surgery, but HCMV infection is not necessary for fibrosis in cardiovascular disease, because the presence of TGF- $\beta 1$ can cause related diseases.

\section{Conclusions}

In summary, this study showed that HCMV could infect HUVECs with EndMT induced by TGF- $\beta 1$. Human cytomegalovirus infection could enhance the autocrine release of TGF- $\beta 1$ in VECs by an MMP cascade reaction. These findings suggested that HCMV-infected arterial endothelial cells may contribute to fibrosis by activating TGF- $\beta 1$, which is involved in cardiovascular disease.

\section{References}

1. Zhang J, Liu YY, Sun HL, et al. High human cytomegalovirus IgG level is associated with increased incidence of diabetic atherosclerosis in type 2 diabetes mellitus patients. Med Sci Monit. 2015;21:4102-4110.

2. Kim JH, Collins-McMillen D, Buehler JC, Goodrum FD, Yurochko AD. Human cytomegalovirus requires epidermal growth factor receptor signaling to enter and initiate the early steps in the establishment of latency in CD34+ human progenitor cells. J Virol. 2017;91(5): e1206-1216.

3. Wang Z, Cai J, Zhang M, et al. Positive expression of human cytomegalovirus phosphoprotein 65 in atherosclerosis. Biomed Res Int. 2016;2016:4067685.

4. Jeong SJ, Han SH, Kim CO, Choi JY, Song YG, Kim JM. Association between human cytomegalovirus antibody levels, and essential hypertension and functional status in elderly Koreans. Geriatr Gerontol Int. 2016;16(1):21-27.

5. Yaiw KC, Mohammad AA, Taher C, et al. Human cytomegalovirus induces upregulation of arginase II: Possible implications for vasculopathies. Basic Res Cardiol. 2014;109(2):401.

6. Yaiw KC, Ovchinnikova O, Taher $\mathrm{C}$, et al. High prevalence of human cytomegalovirus in carotid atherosclerotic plaques obtained from Russian patients undergoing carotid endarterectomy. Herpesviridae. 2013;4(1):3.

7. Maisch T, Kropff B, Sinzger C, Mach M. Upregulation of CD40 expression on endothelial cells infected with human cytomegalovirus. J Virol. 2002;76(24):12803-12812.

8. Rahbar A, Soderberg-Naucler C. Human cytomegalovirus infection of endothelial cells triggers platelet adhesion and aggregation. J Virol. 2005;79(4):2211-2220.

9. Bentz GL, Yurochko AD. Human CMV infection of endothelial cells induces an angiogenic response through viral binding to EGF receptor and beta1 and beta3 integrins. Proc Natl Acad Sci U S A. 2008; 105(14):5531-5536.

10. Delgado JF, Reyne AG, de Dios S, et al. Influence of cytomegalovirus infection in the development of cardiac allograft vasculopathy after heart transplantation. J Heart Lung Transplant. 2015;34(8):1112-1119.

11. Tang-Feldman YJ, Lochhead SR, Lochhead GR, et al. Murine cytomegalovirus (MCMV) infection upregulates P38 MAP kinase in aortas of Apo E KO mice: A molecular mechanism for MCMV-induced acceleration of atherosclerosis. J Cardiovasc Trans/ Res. 2013;6(1):54-64. 
12. Vliegen I, Duijvestijn A, Grauls G, Herngreen S, Bruggeman C, Stassen F. Cytomegalovirus infection aggravates atherogenesis in apoE knockout mice by both local and systemic immune activation. Microbes Infect. 2004;6(1):17-24.

13. Vliegen I, Stassen F, Grauls G, Blok R, Bruggeman C. MCMV infection increases early T-lymphocyte influx in atherosclerotic lesions in apoE knockout mice. J Clin Virol. 2002;25(Suppl 2):S159-171.

14. Hsich E, Zhou YF, Paigen B, Johnson TM, Burnett MS, Epstein SE. Cytomegalovirus infection increases development of atherosclerosis in apolipoprotein-E knockout mice. Atherosclerosis. 2001;156(1): 23-28.

15. Piera-Velazquez S, Mendoza FA, Jimenez SA. Endothelial to mesenchymal transition (EndoMT) in the pathogenesis of human fibrotic diseases. J Clin Med. 2016;5(4). pii:E45. doi:10.3390/jcm5040045

16. Ranchoux B, Antigny F, Rucker-Martin C, et al. Endothelial-to-mesenchymal transition in pulmonary hypertension. Circulation. 2015; 131(11):1006-1018.

17. Good RB, Gilbane AJ, Trinder SL, et al. Endothelial to mesenchymal transition contributes to endothelial dysfunction in pulmonary arterial hypertension. Am J Pathol. 2015;185(7):1850-1858.

18. Chen PY, Qin L, Baeyens N, et al. Endothelial-to-mesenchymal transition drives atherosclerosis progression. J Clin Invest. 2015;125(12): 4514-4528.

19. Wermuth PJ, Li Z, Mendoza FA, Jimenez SA. Stimulation of transforming growth factor-beta1-induced endothelial-to-mesenchymal transition and tissue fibrosis by endothelin-1 (ET-1): A novel profibrotic effect of ET-1. PLoS One. 2016;11(9):e0161988.

20. Yan Y, Wang XJ, Li SQ, et al. Elevated levels of plasma transforming growth factor-beta1 in idiopathic and heritable pulmonary arterial hypertension. Int J Cardiol. 2016;222:368-374.

21. Helantera I, Loginov R, Koskinen P, Tornroth T, Gronhagen-Riska C, Lautenschlager I. Persistent cytomegalovirus infection is associated with increased expression of TGF-beta1, PDGF-AA and ICAM-1 and arterial intimal thickening in kidney allografts. Nephrol Dial Transplant. 2005;20(4):790-796.

22. Annes JP, Munger JS, Rifkin DB. Making sense of latent TGFbeta activation. J Cell Sci. 2003;116(Pt 2):217-224.
23. Tabata T, Kawakatsu H, Maidji E, et al. Induction of an epithelial integrin alphavbeta6 in human cytomegalovirus-infected endothelial cells leads to activation of transforming growth factor-beta1 and increased collagen production. Am J Pathol. 2008;172(4):1127-1140.

24. Abe M, Harpel JG, Metz CN, Nunes I, Loskutoff DJ, Rifkin DB. An assay for transforming growth factor-beta using cells transfected with a plasminogen activator inhibitor-1 promoter-luciferase construct. Anal Biochem. 1994;216(2):276-284.

25. Lin F, Wang N, Zhang TC. The role of endothelial-mesenchymal transition in development and pathological process. IUBMB Life. 2012; 64(9):717-723.

26. Arciniegas E, Neves CY, Carrillo LM, Zambrano EA, Ramirez R. Endothelial-mesenchymal transition occurs during embryonic pulmonary artery development. Endothelium. 2005;12(4):193-200.

27. Zhang Y, Wu X, Li Y, et al. Endothelial to mesenchymal transition contributes to arsenic-trioxide-induced cardiac fibrosis. Sci Rep. 2016;6: 33787.

28. Aisagbonhi O, Rai M, Ryzhov S, Atria N, Feoktistov I, Hatzopoulos AK. Experimental myocardial infarction triggers canonical Wnt signaling and endothelial-to-mesenchymal transition. Dis Model Mech. 2011; 4(4):469-483.

29. Hopper RK, Moonen JR, Diebold I, et al. In pulmonary arterial hypertension, reduced BMPR2 promotes endothelial-to-mesenchymal transition via HMGA1 and its target slug. Circulation. 2016;133(18): 1783-1794.

30. Kong $P$, Christia P, Frangogiannis NG. The pathogenesis of cardiac fibrosis. Cell Mol Life Sci. 2014;71(4):549-574.

31. Catania JM, Chen G, Parrish AR. Role of matrix metalloproteinases in renal pathophysiologies. Am J Physiol Renal Physiol. 2007;292(3):F905-911.

32. Tian YC, Chen YC, Chang CT, et al. Epidermal growth factor and transforming growth factor-beta1 enhance HK-2 cell migration through a synergistic increase of matrix metalloproteinase and sustained activation of ERK signaling pathway. Exp Cell Res. 2007;313(11):2367-2377.

33. Inkinen $\mathrm{K}$, Holma $\mathrm{K}$, Soots $\mathrm{A}$, et al. Expression of TGF-beta and PDGF-AA antigens and corresponding mRNAs in cytomegalovirus-infected rat kidney allografts. Transplant Proc. 2003;35(2):804-805.

34. Streblow DN, Kreklywich CN, Andoh T, et al. The role of angiogenic and wound repair factors during CMV-accelerated transplant vascular sclerosis in rat cardiac transplants. Am J Transplant. 2008;8(2): 277-287. 